\title{
Prospective Study of the Clinical Impact of Epithelial and Mesenchymal Circulating Tumor Cells in Localized Prostate Cancer
}

This article was published in the following Dove Press journal: Cancer Management and Research

\author{
Hailong Liu* \\ Jie Ding* \\ Yanyuan Wu \\ Di Wu \\ Jun Qi
}

Department of Urology, Xinhua Hospital, Shanghai Jiao Tong University School of Medicine, Shanghai 200092, People's Republic of China

*These authors contributed equally to this work
Correspondence: Jun Qi

Department of Urology, Xinhua Hospital, Shanghai Jiao Tong University School of Medicine, No. 1665 Kongjiang Road, Shanghai 200092, People's Republic of China

Email qijun@xinhuamed.com.cn
Background: Although circulating tumor cells (CTCs) are considered as a surrogate marker in monitoring disease progression and treatment response in late stage prostate cancer (PCa), its clinical impact in localized PCa remains unclear, indicating the limitation that is simply based on cell count. This perspective observational study aimed to detect the epithelial-to-mesenchymal transition (EMT) subtypes of CTCs in localized PCa and analyze their clinical relevance and application in predicting PCa stages before surgery compared with the Partin table.

Patients and Methods: Between August 2017 and April 2019, 80 newly diagnosed localized PCa patients were enrolled in the study. Peripheral blood samples $(5 \mathrm{~mL})$ were collected prior to surgery. The CanPatrol ${ }^{\mathrm{TM}} \mathrm{CTC}$ enrichment technique, a size-based isolation method, was used to detect the EMT CTCs. Clinical relevance of the CTCs was analyzed with Spearman's rank correlation test. Models to predict pathological were built with multivariate logistic regression. Receiver operating characteristic (ROC) curve and area under the curve (AUC) analysis were performed to evaluate the accuracy of the prediction model.

Results: CTCs were detected in 55\% of all patients. The biophenotypic CTCs were most valuable and closely correlated with PSA, Gleason score, D'Amico risk classification, and pathological stage in localized PCa. The mesenchymal subtype was rare in this population but associated with seminal vesicle invasion, while the epithelial subtype had limited clinical significance. In addition, the biophenotypic CTCs combined with traditional clinical variables were analyzed by multivariate logistic regression to predict organ-confined disease before surgery, of which the AUC reached 0.818 and was superior to the Partin table 2017 in our cohort. Conclusion: This study highlights the clinical impact of the biophenotypic CTCs in localized PCa, which was most closely related to clinical variables and could help to predict pathology outcomes before surgery.

Keywords: circulating tumor cells, localized prostate cancer, EMT

\section{Introduction}

The incidence of Prostate cancer $(\mathrm{PCa})$ has grown rapidly in China in recent decades. ${ }^{1}$ An accurate classification procedure is critical to distinguish indolent localized PCa from aggressive disease prior to definitive therapy. However, misjudgment of the disease may lead to inappropriate treatment choice. ${ }^{2}$ Currently, treatment options are determined by risk stratification, and several nomograms or statistical models are developed to help predict it before surgery. ${ }^{3}$ One of the most famous and widely used tools is the Partin Table. ${ }^{4}$ However, this nomogram was found not accurate enough when applied in patients out of the US., ${ }^{5,6}$ Thus, 
researchers are considering new clinical parameters, such as the multiparametric MRI, to enhance the predictive accuracy. $^{7}$

Recently, another new clinical parameter, the circulating tumor cells (CTCs), has been applied to clinical oncology gradually. They are malignant cells detached from solid tumor masses and invaded into the bloodstream, which may cause metastatic dissemination. ${ }^{8}$ In its application to PCa, much attention was paid to late stage $\mathrm{PCa}$ or metastatic castration-resistant prostate cancer (mCRPC), partly because of the large amount of CTCs in this stage that was easy to detect. It has been confirmed that CTC counts were closely related to clinical variables. ${ }^{9,10}$ Therefore, CTCs are considered as a surrogate marker even superior to PSA in monitoring disease progression and treatment response in late stage PCa patients. ${ }^{11}$ However, due to technical challenges, the clinical impact of CTCs in localized PCa remains unclear, indicating the limitation that is simply based on cell count. ${ }^{12,13}$ In fact, CTCs could be devided into three subtypes according to the epithelial-mesenchymal transition (EMT) process, which were proved among many cancer types. ${ }^{14}$ EMT was traditionally a basic process in normal physiological events like embryonic development and tissue repair but aberrantly activated in cancerogenesis and metastasis. ${ }^{15}$ Most CTCs originally express epithelial markers, and EMT conveyed mesenchymal and stemness characters to them. ${ }^{16}$ The biophenotypic subtype was a mixture of both epithelial and mesenchymal traits. EMT was once considered an early event in tumor metastasis and progression. ${ }^{17}$ Recently, researches showed that EMT CTCs were not confined to metastatic tumors, but also showed clinical significance in some early stage tumors. A study enrolled 86 early breast cancer patients and detected CTCs in $37.2 \%$ of cases, of which CTCs were correlated with tumor size, stage, molecular classification and preliminarily indicating the association of EMT CTCs and neoadjuvant chemotherapy effectiveness. ${ }^{18}$ In a study with 651 localized colorectal cancer patients, CTCs were found in 535 patients, including 461 cases with the biophenotypic subtype, which associated with clinical stage. ${ }^{19}$ The EMT CTC subtypes also correlated with disease progression and poor prognosis in early stage lung cancer and cervical cancer. ${ }^{20,21}$ Thus, it is conceivable that the EMT CTCs would be useful in the assessment of localized PCa.

There are various techniques to detect CTCs. Among this, the CanPatrol system, developed in 2015, uses the size-based CTC isolation method to enhance the detection rate and could examine multiple biomarkers at the same time. ${ }^{22,23}$ With the filtration-based enrichment method, Awe JA et al found CTCs could be detected in all stages of PCa but did not further evaluate the clinical impact. ${ }^{24}$ Currently, few types of research have studied the CTCs in localized PCa patients with size-based separation method. Studies on the clinical relevance of EMT CTCs in early stage PCa were also limited, with small sample size and simple EMT markers. ${ }^{25}$ The clinical value of EMT CTCs in localized PCa remains to be elucidated.

In this research, we aimed to prospectively identify the EMT subtype CTCs in 80 early stages or localized PCa patients before radical prostatectomy with the size-based isolation method, demonstrate the correlation of different subtypes of CTCs with clinical characteristics, and compare with Partin table 2017 to evaluate the possible predictive value of EMT CTCs in evaluating disease status before surgery.

\section{Patients and Methods Patients and Study Design}

This prospective, single-center study was approved by the Xinhua Hospital Ethics Committee affiliated to Shanghai Jiaotong University School of Medicine and registered in ClinicalTrials.gov (NCT02940977). The trial was conducted in accordance with the Declaration of Helsinki, and all patients provided written informed consent. Between August 2017 and April 2019, patients with newly diagnosed PCa and underwent RP in our hospital were prospectively enrolled in the study cohort. The main inclusion criteria were pathological diagnosis of PCa by biopsy, age $>18$ years, eligible to receive RP, the astern Cooperative Oncology Group (ECOG) performance status of 0 or 1. Patients were excluded if they had other concurrent malignant tumors or severe diseases. Clinical features as age, serum PSA levels, clinical stage, biopsy Gleason score, pathological results were collected. The clinical classification was based on the EAU guidelines of prostate cancer 2017 and D'Amico's classification system for $\mathrm{PCa}^{26}$

\section{Isolation and Enumeration of CTCs with the CanPatrol ${ }^{\mathrm{TM}}$ CTC Assay}

The CanPatrol ${ }^{\mathrm{TM}}$ CTC technique (CTI-Medlab, Shanghai, China, and SurExam, Guangzhou, China) was used for isolating and enumerating CTCs in this study. Detailed protocol has already been published. ${ }^{22,27}$ In short, before surgery, $5 \mathrm{~mL}$ peripheral blood samples were collected in EDTA tubes by venipuncture from selected patients and then transferred into the preserving tubes immediately, stored at $4^{\circ} \mathrm{C}$ for at most 4 
hours before isolation. Peripheral blood samples were treated with erythrocyte lysis buffer to deplete erythrocyte, and washed by PBS with $4 \%$ formaldehyde (Gibco, USA) for 5 min to resuspend the remaining cell mixture. CTCs were isolated with a filtration system, including a $8-\mu \mathrm{m}$ diameter pore calibrated membrane (Millipore, MA, USA), a filtration tube containing the membrane (SurExam, Guangzhou, China), a manifold vacuum plate with valve settings (SurExam, Guangzhou, China), an E-Z 96 vacuum manifold (Omega, GA, USA), and a vacuum pump (SurExam, Guangzhou, China). The EMT subtypes were classified by the epithelial and mesenchymal markers expressed in CTCs with a multiplex RNA-in situ hybridization (RNA-ISH) assay. EMT markers were categorized into 3 groups as previously described. ${ }^{28}$ Group 1 contained four epithelial biomarkers (EpCAM, Cytokeratin 8/18/19), Group 2 contained two mesenchymal biomarkers (Vimentin and Twist), Group 3 contained CD45 biomarker to remove leukocytes from CTCs. Finally, cell nucleus was visualized with DAPI for 5 minutes (Sigma, St. Louis, USA). The processed cells were analyzed with an automated imaging fluorescent microscope (Zeiss, Germany). The red and green fluorescent dots emitted by cells presented epithelial and mesenchymal markers, respectively. The white dots represented CD45 expression of white blood cells.

\section{Statistical Analysis}

All statistical analyses were performed with the statistical package for SPSS 19.0 (SPSS Inc. Chicago, IL). Clinical relevance of the CTC parameters was analyzed with Spearman's rank correlation test. Univariate and multivariate logistic regression were performed to analyze independent factors. Models to predict pathological were built with multivariate logistic regression. Receiver operating characteristic (ROC) curve and area under the curve (AUC) analysis was performed to evaluate the accuracy of the prediction model. All statistical tests were two-tailed. A P value $<0.05$ was considered statistically significant.

\section{Results}

\section{Baseline Characteristics of the Study Population and Comparison with Partin Table 2017}

Eighty localized PCa patients were enrolled in our cohort. Clinical variables of the current study are summarized in Table 1 . To test the accuracy of the Partin table in our cohort, patients from the Partin table 2017 were compared to the control group. ${ }^{4}$ Generally, there were some differences in
Table I Baseline Characteristics of the Study Population and Comparison with Partin Table 2017

\begin{tabular}{|c|c|c|}
\hline Characteristics & Current Study & Partin 2017 \\
\hline Patients, $\mathrm{n}$ & 80 & 5629 \\
\hline \multicolumn{3}{|l|}{ Age, years } \\
\hline Mean (SD) & $68.58(6.75)$ & $59.5(7.0)$ \\
\hline \multicolumn{3}{|l|}{ Clinical stage, n (\%) } \\
\hline TIc & $5(6)$ & $3514(79)$ \\
\hline $\mathrm{T} 2 \mathrm{a}$ & $37(46)$ & $651(15)$ \\
\hline $\mathrm{T} 2 \mathrm{~b}$ & II (14) & $243(5)$ \\
\hline $\mathrm{T} 2 \mathrm{c}$ & $23(29)$ & $5 I(I)$ \\
\hline T3a* & $4(5)$ & \\
\hline \multicolumn{3}{|l|}{ PSA (ng/mL), n (\%) } \\
\hline $0-2.5$ & $2(2.5)$ & $760(17)$ \\
\hline $2.6-4.0$ & $2(2.5)$ & $680(15)$ \\
\hline $4.1-6.0$ & $6(7.5)$ & $1584(36)$ \\
\hline $6.1-10.0$ & $24(30)$ & $1038(23)$ \\
\hline$>10.0$ & $46(57.5)$ & $397(9)$ \\
\hline \multicolumn{3}{|l|}{ Biopsy Gleason score, n (\%) } \\
\hline$\leq 6(\mathrm{GGI})$ & $16(20)$ & $1617(36)$ \\
\hline $3+4(G G 2)$ & $24(30)$ & $1687(38)$ \\
\hline $4+3(G G 3)$ & $24(30)$ & $664(15)$ \\
\hline 8 (GG4) & $9(\mathrm{II})$ & $211(5)$ \\
\hline 9-10 (GG5) & $7(9)$ & $271(6)$ \\
\hline \multicolumn{3}{|l|}{ Pathological stage } \\
\hline OC & $50(62.5)$ & $3278(74)$ \\
\hline EPE (SVI-, LNI-) & $19(24)$ & $898(20)$ \\
\hline SVI (LNI-) & $10(12.5)$ & $182(4)$ \\
\hline LNI & $\mathrm{I}(\mathrm{I})$ & $101(2)$ \\
\hline
\end{tabular}

Note: *Patients of T3a were excluded in the comparison with Partin table. Abbreviations: OC, organ-confined; EPE, extracapsular extension; SVI, seminal vesicle invasion; $\mathrm{LNI}$, lymph node invasion.

clinical distribution of the two cohorts. The average age in our cohort was $68.58 \pm 6.75$ years, which was higher than that in Partin table 2017 (59.5 7.0 years). There was also difference in clinical stage distribution. In our study, stage T2a (46\%) was common, while in Partin table 2017, most were from stage T1c (79\%). Serum PSA distribution of the two groups was not equally comparable. More than half of the patients in our study had serum PSA values higher than $10 \mathrm{ng} / \mathrm{mL}$, but PSA in the other cohort mostly ranged from 4 to $10 \mathrm{ng} / \mathrm{mL}$. The distribution of biopsy Gleason score and pathological stage was similar in each group.

The accuracy of the Partin Table 2017 predictions in our cohort was quantified with ROC derived AUC estimates, where the predicted organ-confined (OC), extracapsular extension (EPE), seminal vesicle invasion (SVI), and lymph node invasion (LNI) are compared with actual 
pathological characteristics (Figure 1). As the 2017 Partin table can only predict patients with stage before $\mathrm{T} 2 \mathrm{c}$, the four patients of T3a in our cohort were excluded in the comparison analysis. The AUC of the 2017 Partin Table's OC, EPE, SVI, and LNI was $0.735(\mathrm{p}<0.001,95 \%$ CI $0.623-0.847$, Figure $1 \mathrm{~A}), 0.578$ ( $\mathrm{p}=0.301,95 \%$ CI $0.436-$ 0.719 , Figure 1B), $0.883(\mathrm{p}<0.001,95 \%$ CI $0.797-0.968$, Figure $1 \mathrm{C})$, and $0.873(\mathrm{p}=0.201,95 \%$ CI $0.75-0.997$, Figure 1D) respectively, suggesting a relatively poor predictive value on OC and EPE.

\section{Detection of CTCs and Positivity with Clinical Characteristics}

Of all the 80 patients, 44 (55\%) cases could be detected with CTCs, ranging from 1 to 13 per $5 \mathrm{~mL}$. Representative images of the three subtypes of CTCs are shown in Figure 2. Among

A

A $O C$

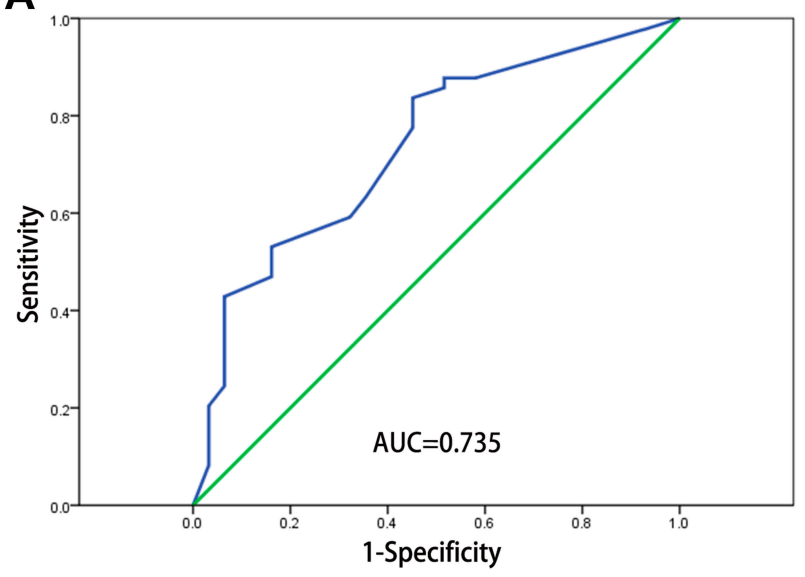

C

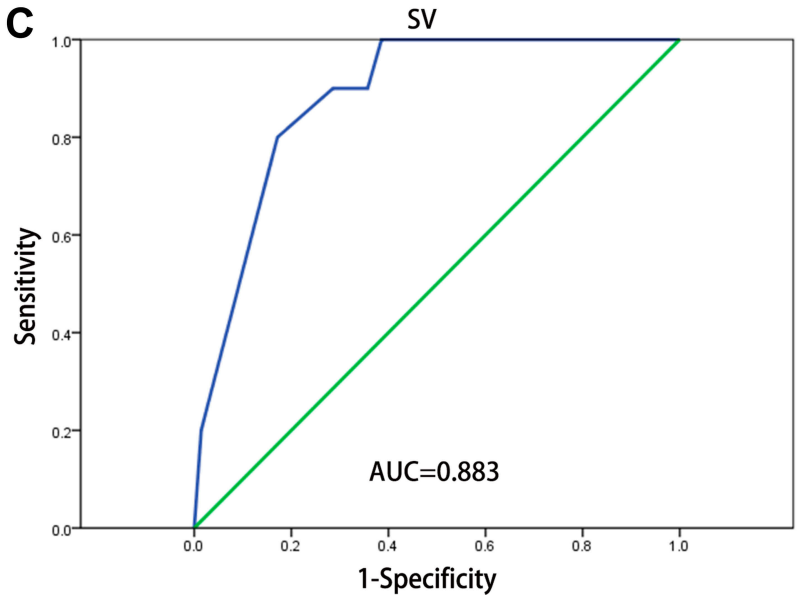

all the three subtypes, the biophenotypic CTCs were the most common, with 38 patients detected, while epithelial and mesenchymal CTCs accounted for $38.8 \%$ (31/80) and $12.5 \%(10 / 80)$ respectively. Seven patients were found to have all the three CTC subtypes. Detailed distribution is displayed in Table 2. Except for the mesenchymal CTCs, the other two CTC subtypes and total CTCs had a higher propensity to be detected in patients with higher clinical stage, pathological stage, and D'Amico risk classification, but not closely related to age, PSA levels, or Gleason score.

\section{Association of CTCs Counts with Clinical Characteristics}

The number of CTCs was further assessed with the clinical features. The average amount of epithelial CTCs in each pathological stage was 0.71 (range $0-4$ ), 0 (range $0-0$ ),

B

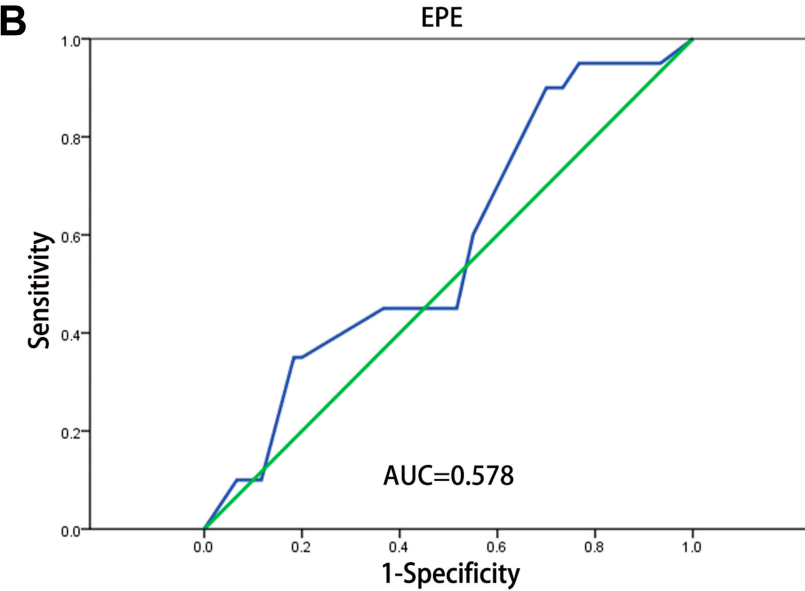

D

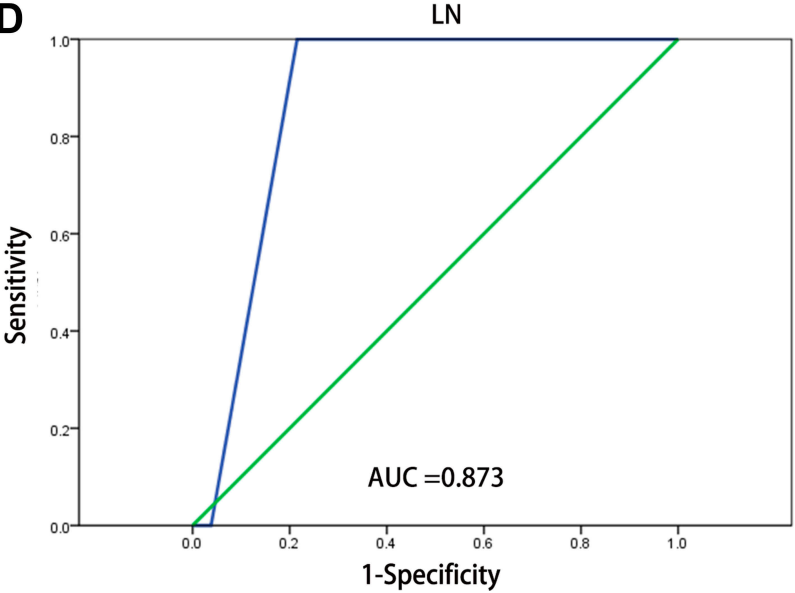

Figure I ROC curve analysis on the Partin table 2017 in our cohort. (A-D) ROC curves for OC, organ-confined; EPE, extracapsular extension; SV, seminal vesicle invasion; LN, lymph node invasion.

Abbreviations: ROC, receiver operating characteristic; AUC, area under the curve. 

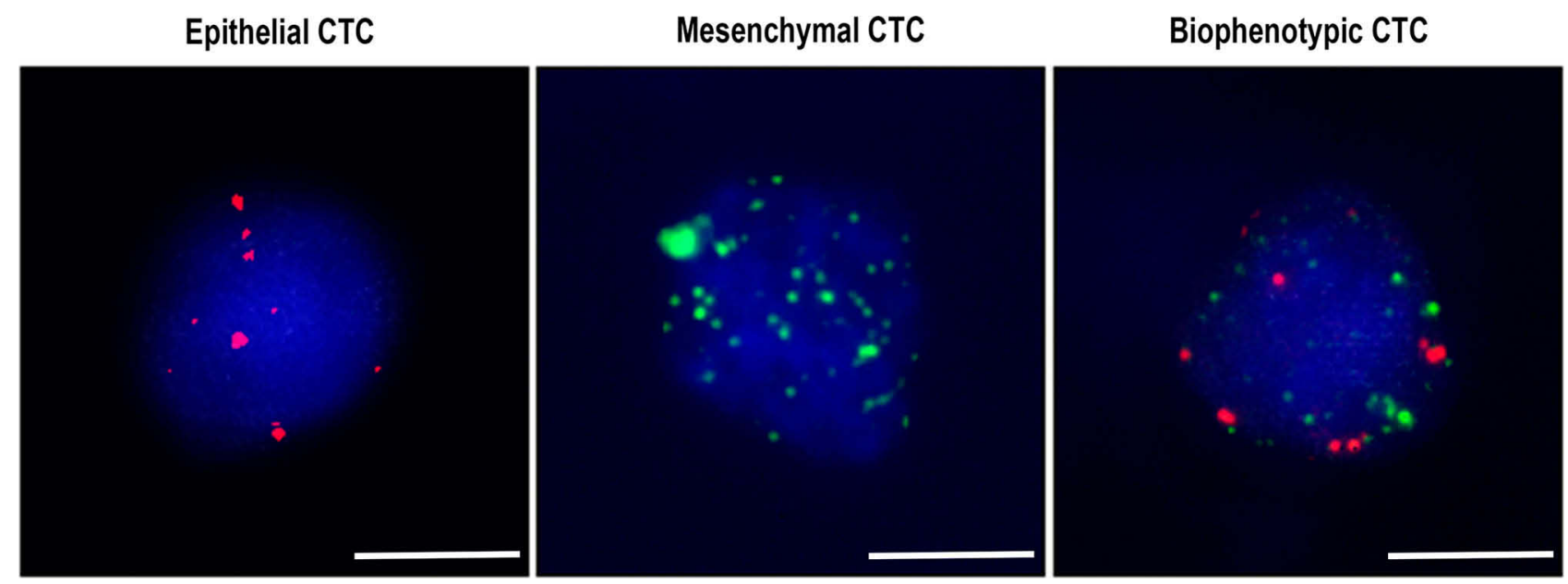

Figure 2 Representative images of the three subtypes of CTCs in PCa patients. Epithelial CTC (left): stained with only epithelial molecular markers (red dots), mesenchymal CTC (middle): stained with only mesenchymal molecular markers (green dots), and biophenotypic CTC (right): stained with both epithelial and mesenchymal molecular markers (red and green dots). Bars $=5 \mu \mathrm{m}$.

0.54 (range 0-2), 1.18 (range 0-6), 1.43 (range 0-4) and 4 (range 4-4), respectively. The amount of biophenotypic CTCs was 0.38 (range 0-4), 1.67 (range 0-5), 0.88 (range 0-4), 2.41 (range 0-8), 3.57 (range 1-6), and 7 (range 7-7), respectively. Few mesenchymal CTCs were detected in each stage (Table S1). Spearman's rank correlation test indicated that the biophenotypic subtype had the most significant correlation with almost all clinical variables, except age and lymph node invasion (Table 3). However, its connection with PSA and Gleason score was relatively low ( $\rho=0.247,0.259$, respectively). When we divided serum PSA levels and Gleason score into low and high groups ( $\mathrm{PSA} \geq 20 \mathrm{ng} / \mathrm{mL}, \mathrm{GS} \geq 4+3$ ), there was no correlation with any CTC counts (Figure 3A and C). Both pathological stage and D'Amico risk classification had a strong association with the amount of total and biophenotypic CTCs (Table 3, Figure 3B and D). However, no significant correlation emerged between pathological stage and the epithelial subtype. In fact, epithelial CTCs were not associated with most variables, but only had a slight correlation with D'Amico risk classification and OC ( $\rho=0.222,-0.236$, respectively). The OC, EPE, and SVI were extended expansion from pathological stage. As a result, OC was also found negatively related to the amount of total and biophenotypic CTCs, and EPE and SVI had a positive relationship. The mesenchymal subtype was detected in 10 patients. When taken into analysis, this subtype was only a hint for seminal vesicle invasion. None of the three CTC subtypes were related to age or lymph node invasion. It was worth noting that there was only one lymph node positive patients in our cohort, correlation analyses of lymph node invasion may be inaccurate. In sum, these results suggest the presence of biophenotypic CTCs most closely correlates with disease severity.

\section{EMT Subtypes of CTCs Help to Predict Pathological Outcomes in Prostate Cancer}

As EMT CTCs, especially the biophenotypic subtype, were found closely associated with clinical characteristics, multivariable logistic regression was analyzed to find the potential risk factors. Asthe value of Partin table 2017 to predict OC and EPE in our PCa cohort was limited (Figure 1), we try to build models with CTCs to predict PCa in OC and EPE. The AUC was calculated to assess the accuracy of these regression models. As a result, the number of the biophenotypic CTCs and clinical stage were negatively correlated with $\mathrm{OC}$ in $\mathrm{PCa}(\mathrm{p}=0.01, \mathrm{OR}=0.582$ and $\mathrm{p}=0.016, \mathrm{OR}=0.527$, respectively, Table 4). The AUC of the predictive model for OC was $0.818(\mathrm{p}<0.001,95 \% \mathrm{CI}$ 0.726-0.909), which was higher than clinical stage (AUC $=$ 0.698, $\mathrm{p}=0.003,95 \%$ CI 0.582-0.815), CTC (AUC= $0.788, \mathrm{p}<0.001,95 \%$ CI $0.683-0.893$ ), or Partin table alone $(\mathrm{AUC}=0.735, \mathrm{p}<0.001,95 \%$ CI $0.623-0.847)$ (Table 4, Figure 4A). In prediction of EPE, only the biophenotypic CTCs were statistically significant $(\mathrm{p}<0.01, \quad \mathrm{OR}=1.383$, Table 5). The AUC was 0.72 $(p=0.003,95 \%$ CI $0.592-0.847$, Table 5, Figure 4B). As to seminal vesicle invasion, influence factors included the mesenchymal CTCs subtype, pathological stage, and 
Table 2 Baseline Characteristics of the Study Population and CTCs According to Different Subgroups

\begin{tabular}{|c|c|c|c|c|c|c|c|c|c|c|c|c|}
\hline \multirow[t]{2}{*}{ Characteristics } & \multicolumn{2}{|c|}{ Total CTCs } & \multirow[t]{2}{*}{$\mathbf{P}$} & \multicolumn{2}{|c|}{ Epithelial CTCs } & \multirow[t]{2}{*}{$\mathbf{P}$} & \multicolumn{2}{|c|}{ Biophenotypic CTCs } & \multirow[t]{2}{*}{$\mathbf{P}$} & \multicolumn{2}{|c|}{ Mesenchymal CTCs } & \multirow[b]{2}{*}{$\mathbf{P}$} \\
\hline & Positive & Negative & & Positive & Negative & & Positive & Negative & & Positive & Negative & \\
\hline Patients, n(\%) & $44(55)$ & $36(45)$ & & $31(38.8)$ & $49(61.2)$ & & $38(47.5)$ & $42(52.5)$ & & $10(12.5)$ & $70(87.5)$ & \\
\hline Age, years & & & 0.81 & & & 0.79 & & & 0.87 & & & 0.97 \\
\hline Mean (SD) & $68.4(6.5)$ & $68.8(7.1)$ & & $68.3(7.6)$ & $68.7(6.2)$ & & $68.7(6.5)$ & $68.5(7.0)$ & & $68.6(5.5)$ & $68.6(6.9)$ & \\
\hline Clinical stage, $n(\%)$ & & & 0.02 & & & 0.04 & & & 0.01 & & & 0.41 \\
\hline T Ic & $2(2.5)$ & $3(3.8)$ & & $2(2.5)$ & $3(3.8)$ & & $\mathrm{I}(\mathrm{I} .3)$ & $4(5)$ & & $0(0)$ & $5(6.3)$ & \\
\hline T 2a & $16(20)$ & $21(26.3)$ & & $9(11.3)$ & $28(35)$ & & $14(17.5)$ & $23(28.6)$ & & $4(5)$ & $33(41.3)$ & \\
\hline $\mathrm{T} 2 \mathrm{~b}$ & $7(8.8)$ & $4(5)$ & & $6(7.5)$ & $5(6.3)$ & & $6(7.5)$ & $5(6.3)$ & & $2(2.5)$ & $9(11.3)$ & \\
\hline T 2c & $16(20)$ & $7(8.8)$ & & $12(15)$ & $11(13.8)$ & & |4(I7L.5) & $9(11.3)$ & & $4(5)$ & $19(23.8)$ & \\
\hline T 3a & $3(3.8)$ & $\mathrm{I}(1.3)$ & & $2(2.5)$ & $2(2.5)$ & & $3(3.8)$ & $\mathrm{I}(1.3)$ & & $0(0)$ & $4(5)$ & \\
\hline PSA (ng/mL) & & & 0.58 & & & 0.10 & & & 0.06 & & & 0.62 \\
\hline Mean (SD) & $24.6(31.8)$ & $13.8(12.1)$ & & $25.5(36.6)$ & $16.1(13.7)$ & & $21.2(32.8)$ & $15.4(14.5)$ & & $18.8(13.6)$ & $19.9(26.7)$ & \\
\hline $\begin{array}{l}\text { Biopsy Gleason score, } \\
n(\%)\end{array}$ & & & 0.30 & & & 0.34 & & & 0.08 & & & 0.12 \\
\hline$\leq 6(\mathrm{GGI})$ & $8(10)$ & $8(10)$ & & $6(7.5)$ & $10(12.5)$ & & $5(6.3)$ & $11(13.8)$ & & $2(2.5)$ & $14(17.5)$ & \\
\hline $3+4(\mathrm{GG} 2)$ & $13(16.3)$ & $11(13.8)$ & & $8(10)$ & $16(20)$ & & $12(15)$ & $12(15)$ & & $0(0)$ & $24(30)$ & \\
\hline $4+3(\mathrm{GG} 3)$ & $12(15)$ & $12(15)$ & & $9(11.3)$ & $15(18.8)$ & & $11(13.8)$ & $13(16.3)$ & & $4(5)$ & $20(25)$ & \\
\hline 8 (GG4) & $4(5)$ & $5(6.3)$ & & $2(2.5)$ & $7(8.8)$ & & $3(3.8)$ & $6(7.5)$ & & $\mathrm{I}(1.3)$ & $8(10)$ & \\
\hline $9-10$ (GG5) & $7(8.8)$ & $0(0)$ & & $6(7.5)$ & $\mathrm{I}(1.3)$ & & $7(8.8)$ & $0(0)$ & & $3(3.8)$ & $4(5)$ & \\
\hline $\begin{array}{l}\text { D’Amico risk } \\
\text { classification, } n(\%)\end{array}$ & & & 0.01 & & & 0.01 & & & 0.00 & & & 0.07 \\
\hline Low & $2(2.5)$ & $3(3.8)$ & & $\mathrm{I}(1.3)$ & $4(5)$ & & $\mathrm{I}(\mathrm{I} .3)$ & $4(5)$ & & $0(0)$ & $5(6.3)$ & \\
\hline Medium & $11(13.8)$ & $18(22.5)$ & & $7(8.8)$ & $22(27.5)$ & & $9(\mid 1.3)$ & $20(25)$ & & $2(2.5)$ & $27(33.8)$ & \\
\hline High & $3 \mathrm{I}(38.8)$ & $15(18.6)$ & & $23(28.8)$ & $23(28.8)$ & & $28(35)$ & $18(22.5)$ & & $8(10)$ & $38(47.5)$ & \\
\hline Pathological stage, n(\%) & & & 0.00 & & & 0.08 & & & 0.00 & & & 0.07 \\
\hline PT2a & $7(8.8)$ & $14(17.5)$ & & $7(8.8)$ & $14(17.5)$ & & $4(5)$ & $17(21.3)$ & & $2(2.5)$ & $19(23.8)$ & \\
\hline $\mathrm{pT} 2 \mathrm{~b}$ & $\mathrm{I}(\mathrm{I} .3)$ & $2(2.5)$ & & $0(0)$ & $3(3.8)$ & & $\mathrm{I}(\mathrm{I} .3)$ & $2(2.5)$ & & $0(0)$ & $3(3.8)$ & \\
\hline $\mathrm{pT} 2 \mathrm{c}$ & $11(13.8)$ & $15(18.8)$ & & $9(\mid 1.3)$ & $17(21.3)$ & & $9(\mid 1.3)$ & $17(21.3)$ & & $I(1.3)$ & $25(31.3)$ & \\
\hline рT3a & $17(21.3)$ & $5(6.3)$ & & $9(11.3)$ & $13(16.3)$ & & $16(20)$ & $6(7.5)$ & & $3(3.8)$ & $19(23.8)$ & \\
\hline pT3b & $7(8.8)$ & $0(0)$ & & $5(6.3)$ & $2(2.5)$ & & $7(8.8)$ & $0(0)$ & & $3(3.8)$ & $4(5)$ & \\
\hline pT4 & $I(1.3)$ & $0(0)$ & & $\mathrm{I}(1.3)$ & $0(0)$ & & $I(1.3)$ & $0(0)$ & & $\mathrm{I}(1.3)$ & $0(0)$ & \\
\hline $\begin{array}{l}\text { Prostatectomy } \\
\text { Gleason score, n(\%) }\end{array}$ & & & 0.22 & & & 0.72 & & & 0.052 & & & 0.76 \\
\hline$\leq 6(\mathrm{GGI})$ & $4(5)$ & $3(3.8)$ & & $3(3.8)$ & $4(5)$ & & $2(2.5)$ & $5(6.3)$ & & $2(2.5)$ & $5(6.3)$ & \\
\hline $3+4(\mathrm{GG} 2)$ & $14(17.5)$ & $13(16.3)$ & & $12(15)$ & $15(18.8)$ & & $12(15)$ & $15(18.8)$ & & $2(2.5)$ & $25(31.3)$ & \\
\hline $4+3(G G 3)$ & $15(18.8)$ & $18(22.5)$ & & $10(12.5)$ & $23(28.8)$ & & $14(17.5)$ & $19(23.8)$ & & $3(3.8)$ & $30(37.5)$ & \\
\hline 8 (GG4) & $\mathrm{I}(1.3)$ & $2(2.5)$ & & $\mathrm{I}(1.3)$ & $2(2.5)$ & & $\mathrm{I}(1.3)$ & $2(2.5)$ & & $0(0)$ & $3(3.8)$ & \\
\hline 9-10 (GG5) & $10(12.5)$ & $0(0)$ & & $5(6.3)$ & $5(6.3)$ & & $9(11.3)$ & $I(1.3)$ & & $3(3.8)$ & $7(8.8)$ & \\
\hline
\end{tabular}

Notes: Positive: patients with I or more CTCs detected; Negative: patients with no CTCs detected. Gamma test. P value $<0.05$ is highlighted in bold.

biopsy Gleason score $(\mathrm{p}=0.011, \mathrm{OR}=4.353, \mathrm{p}=0.027$, was positive in our cohort and the low detection rate may $\mathrm{OR}=2.875$, and $\mathrm{p}=0.038, \mathrm{OR}=2.224$, respectively, not represent the whole population (data not shown). Table S2). No items were related to lymph node invasion These results further verified the application value of in the final logistic regression analysis, as only one case EMT CTCs in localized PCa, suggesting the 
Table 3 Correlation Between Clinical Variables and CTCs Number

\begin{tabular}{|l|l|l|l|l|}
\hline \multirow{2}{*}{ Characteristics } & \multicolumn{2}{l}{$\boldsymbol{\rho}$} \\
\cline { 2 - 5 } & Total CTCs & Epithelial CTCs & \multicolumn{1}{l|}{ Biophenotypic CTCs } & Mesenchymal CTCs \\
\hline Age & -0.001 & -0.04 & 0.005 & -0.027 \\
PSA & 0.209 & 0.062 & $0.247^{*}$ & 0.038 \\
Biopsy Gleason score & 0.212 & 0.135 & $0.259^{*}$ & 0.193 \\
D'Amico risk classification & $0.303^{* *}$ & $0.222^{*}$ & $0.33^{* *}$ & 0.172 \\
Pathological stage & $0.45 I^{* *}$ & 0.196 & $0.527^{* *}$ & $0.239 *$ \\
Prostatectomy Gleason score & 0.18 & -0.025 & $0.29 * *$ & 0.026 \\
OC & $-0.478^{* *}$ & $-0.236 *$ & $-0.527^{* *}$ & -0.227 \\
EPE (SV-, LN-) & $0.299 * *$ & 0.12 & $0.357^{* *}$ & -0.57 \\
SVI & $0.313^{* *}$ & 0.163 & $0.343^{* *}$ & $0.423^{* *}$ \\
LNI & -0.005 & 0.081 & -0.1 & -0.042 \\
\hline
\end{tabular}

Notes: Spearman's rank correlation; $* \mathrm{P}<0.05$, ${ }^{*} \mathrm{P}<0.0 \mathrm{I} ; \rho$, correlation coefficient.

Abbreviations: OC, organ-confined; EPE, extracapsular extension; SVI, seminal vesicle invasion; LNI, lymph node invasion.

biophenotypic subtype was an independent factor and help to predict OC and EPE, and the mesenchymal subtype was an independent factor of SVI.

\section{Discussion}

In the present study, we verified the limited application value of Partin table 2017 in a Chinese cohort, identified CTCs classification with EMT markers in localized PCa. We also evaluated the clinical significance of these EMT subtypes and found EMT CTCs could add value to traditional parameters in predicting postoperative pathological stage, indicating a valuable role in localized PCa prediction.

The Partin Tables intended to predict pathologic stage before RP and help clinicians make a surgical decision. ${ }^{29}$ But several studies verified previous Partin Tables were not accurate out of the US. Bhojani et al reevaluated the 2007 Partin Tables on 3105 patients treated with RP at a single-center cohort in European institution and found the accuracy of predicted probabilities to observed rates was poor. ${ }^{6}$ Another study assessed three versions of Partin table in the Chinese population, finding the AUC of organ-confined disease (OC) was $0.877,0.788$, and 0.726 , respectively, and EPE prediction was even lower. ${ }^{30}$ The possible reason was the different distribution of clinical characters in these cohorts. ${ }^{31}$ The Partin table 2017 is the fifth edition, while few researchers have evaluated its accuracy. In our study, 76 patients within stage T2c were joined in the comparison. The prediction value on OC and EPE was limited, with the AUC of 0.735 (Figure 1A) and 0.578 (Figure 1B) respectively, which means nearly half of the patients with EPE would be inaccurately evaluated. Misestimate may lead to inappropriate treatment choices such as preservation of the neurovascular bundle in operation. Thus, there is a need for new parameters to help increase the accuracy in predicting pathological stage of $\mathrm{PCa}$.

CTC detection, liquid biopsy from the peripheral blood of cancer patients, has been regarded a promising technique for cancer diagnosis and prognostic analysis in recent years. $^{32}$ However, due to the low detection rate and unstable results, its utility in localized $\mathrm{PCa}$ remains unclear. In the past decades, the amount of CTCs in early stage $\mathrm{PCa}$ was determined by distinct equipment and methods used for collection or detection. In the 1990s, CTCs were discovered by RT-PCR to target the mRNA of PSA or PSMA in blood. ${ }^{33-35}$ But results were inconsistent, because of the different primers used for RTPCR and various methodological treatments. Besides, this method only tests the relative mRNA amount of target genes and could not count and isolate CTCs from blood. $^{25}$ Since 2008, the CellSearch system has been applied to detect CTCs in PCa. ${ }^{13}$ However, this methodology may underestimate the actual counts because of the fragmentation of fragile CTCs and enrichment of cells by the EpCAM marker on the cell surface. Cells with epithelial-to-mesenchymal transition and no longer express epithelial markers may escape from this EpCAM-based detection. ${ }^{36}$ Generally, detection rate of this method was less than $15 \%$ in localized $\mathrm{PCa}$, with no clinical significance. $^{37-39}$ A few studies tried to add some 2 times more blood samples for detection, increasing the positive rate up to $49 \%$, but still could not find clinical impact of these CTCs. ${ }^{40}$ As a result, the low sensitivity and specificity limited its application in early stage PCa. ${ }^{12,13}$ 
A

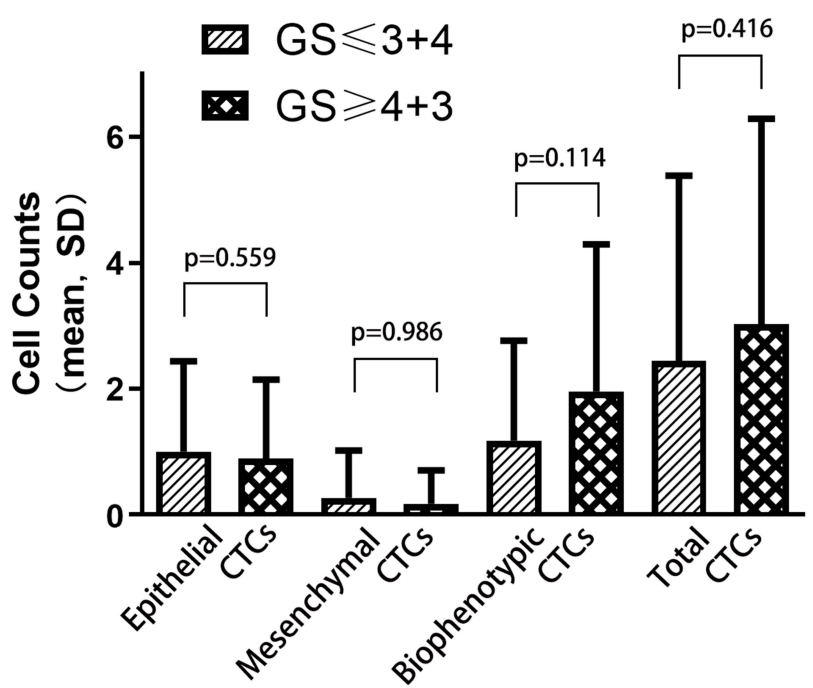

C

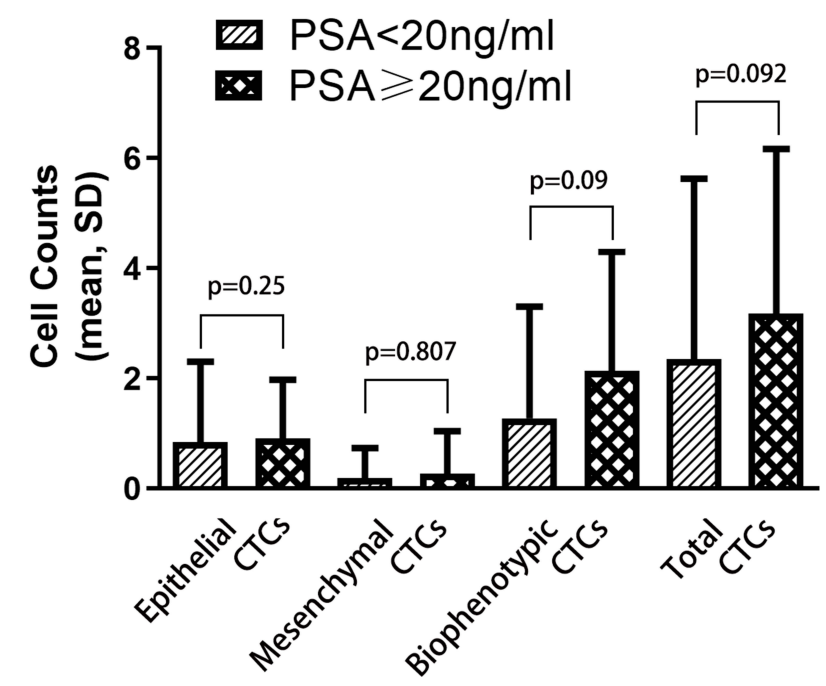

B

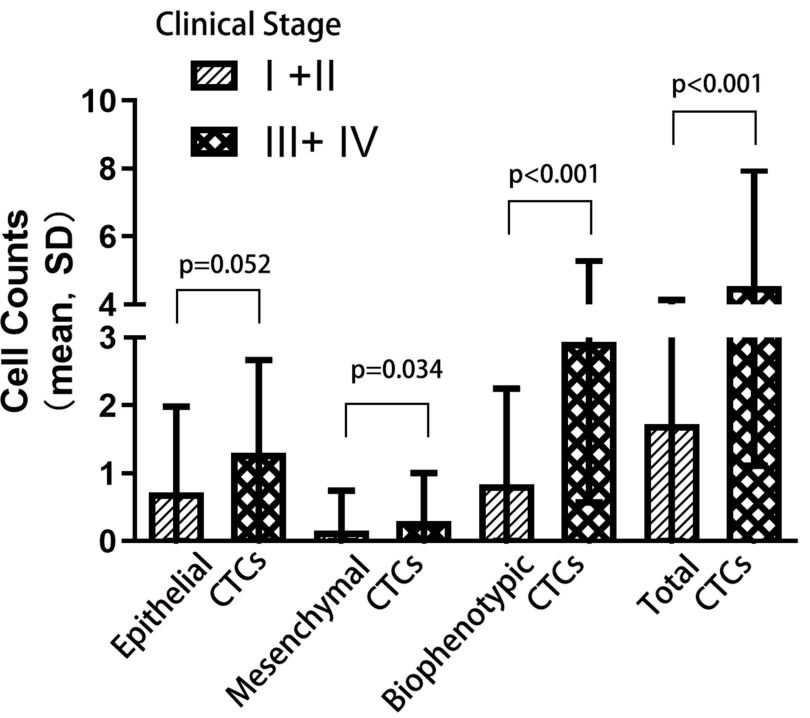

D

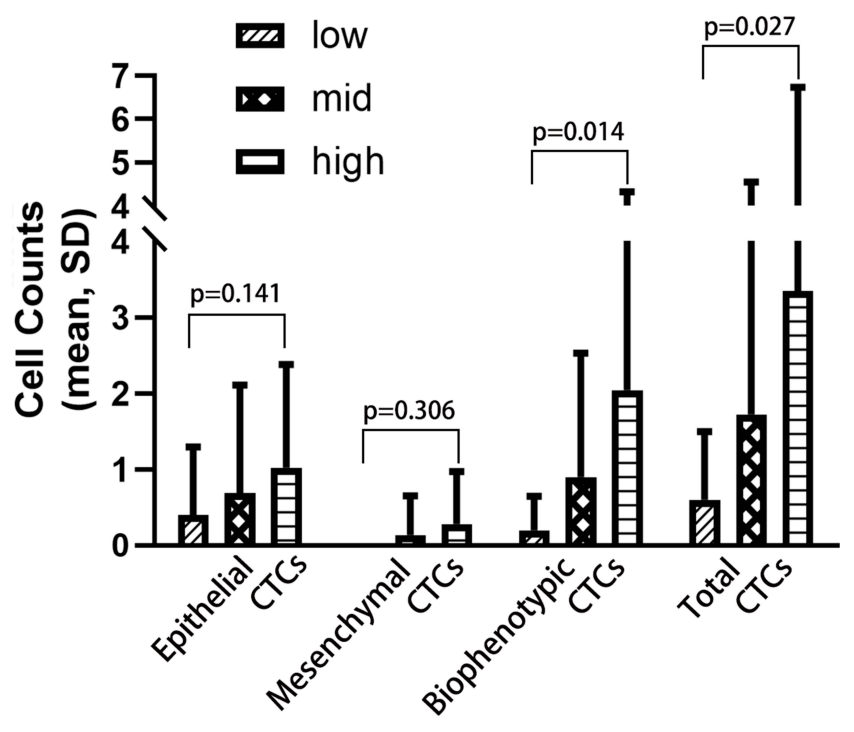

Figure 3 The correlation EMT CTC counts with clinical variables. Association of epithelial, biophenotypic, and mesenchymal CTCs with (A) Gleason score, (B) clinical stage, (C) PSA, (D) D'Amico risk classification, analyzed by Mann-Whitney U-test or Kruskal-Wallis $H$-test.

In our study, detection rate of total CTCs reached $55 \%$ (Table 2), because we adopted another detection technique, of which CTCs were isolated by cell size and deformability. This detection method simplifies the filter progress, decreasing the loss of CTCs caused by multiple centrifugations and elution. Meanwhile, multiple epithelial and mesenchymal RNA-ISH markers were adopted to distinguish the isolated CTCs, increasing the detection sensitivity. ${ }^{22}$ With this method, a small-scale study on 38 localized PCa patients found that the positivity of CTC reached $63 \%$ and tend to be correlated with high-risk classification, which needs validation of in a larger sample count. ${ }^{41}$ Meanwhile, this study diagnosed EMT CTCs with only two markers, cytokeratin for the epithelial subtype and vimentin for the mesenchymal subtype, respectively, which may decrease the detection rate. Our study, with a larger cohort of 80 localized PCa patients, verified this assumption. In our study, CTC positive rate was proved associated with highrisk classification and pathological stage (Table 2).

In CRPC patients, the biophenotypic CTCs were found to have a better prognostic value than the epithelial subtype. ${ }^{42}$ Another study on high-risk PCa found that in CTCs the EMT 
Table 4 Logistic Regression Analysis of OC

\begin{tabular}{|c|c|c|c|c|c|}
\hline \multirow[t]{2}{*}{ Characteristics } & \multicolumn{2}{|c|}{ Univariate Logistic Regression } & \multirow[t]{2}{*}{ B } & \multicolumn{2}{|c|}{ Multivariate Logistic Regression } \\
\hline & HR $(95 \% \mathrm{Cl})$ & $P$ value & & HR (95\% Cl) & $\mathbf{P}$ \\
\hline Age, & $0.988(0.924-1.057)$ & 0.728 & & & \\
\hline Clinical stage & $0.479(0.30 \mathrm{I}-0.763)$ & 0.002 & -0.64 & $0.527(0.313-0.887)$ & 0.016 \\
\hline PSA level & $0.967(0.936-0.998)$ & 0.037 & & - & 0.353 \\
\hline Prostate volume & $0.999(0.976-1.022)$ & 0.900 & & & \\
\hline Biopsy Gleason score & $0.714(0.483-1.057)$ & 0.092 & & & \\
\hline D'Amico risk classification & $0.379(0.16 \mathrm{I}-0.892)$ & 0.026 & & - & 0.602 \\
\hline Total CTCs & $0.715(0.598-0.854)$ & 0.000 & & - & 0.529 \\
\hline Epithelial CTCs & $0.654(0.454-0.942)$ & 0022 & & - & 0.623 \\
\hline Biophenotypic CTCs & $0.57(0.426-0.762)$ & 0.000 & -0.542 & $0.582(0.428-0.790)$ & 0.001 \\
\hline Mesenchymal CTCs & $0.62(0.293-1.3 \mid 4)$ & 0.212 & & & \\
\hline
\end{tabular}

Note: $P$ value $<0.05$ is highlighted in bold.

Abbreviation: OC, organ-confined disease.

markers were more common than the epithelial markers. In metastatic castration-sensitive PCa, EMT CTCs at baseline predicted the effective time of standard $\mathrm{ADT}^{43}$ Our study focused on localized PCa patients, suggesting the number of biophenotypic CTCs was closely related to a series of clinical variables (Table 3). In the logistic regression analysis on OC, biophenotypic CTCs and clinical stage were the only two significant factors, with similar hazard ratio, which means this mixed subtype CTCs were as important as clinical stage. The biophenotypic subtype was once proposed to be a transient subtype. ${ }^{44}$ But recent studies showed this phenotype was quite stable as it had both the epithelial and mesenchymal plasticity. ${ }^{45,46}$ Because the biophenotypic phenotype was found more stem-like than the complete mesenchymal subtype, ${ }^{47}$ this mixed phenotype has several advantages in survival over the epithelial or mesenchymal subtypes under stress conditions, which maybe the reason of the high ratio of biophenotypic subtype CTCs in our study. ${ }^{48,49}$ It was also noticeable that the biophenotypic phenotype was more common when isolating EMT CTCs by cell size, suggesting these results may partly be method-dependent. ${ }^{19,41}$ In sum, the biophenotypic CTCs were the most valuable subtypes related to localized PCa. It showed great potential as a biomarker for judgment of disease status. ${ }^{50}$
A

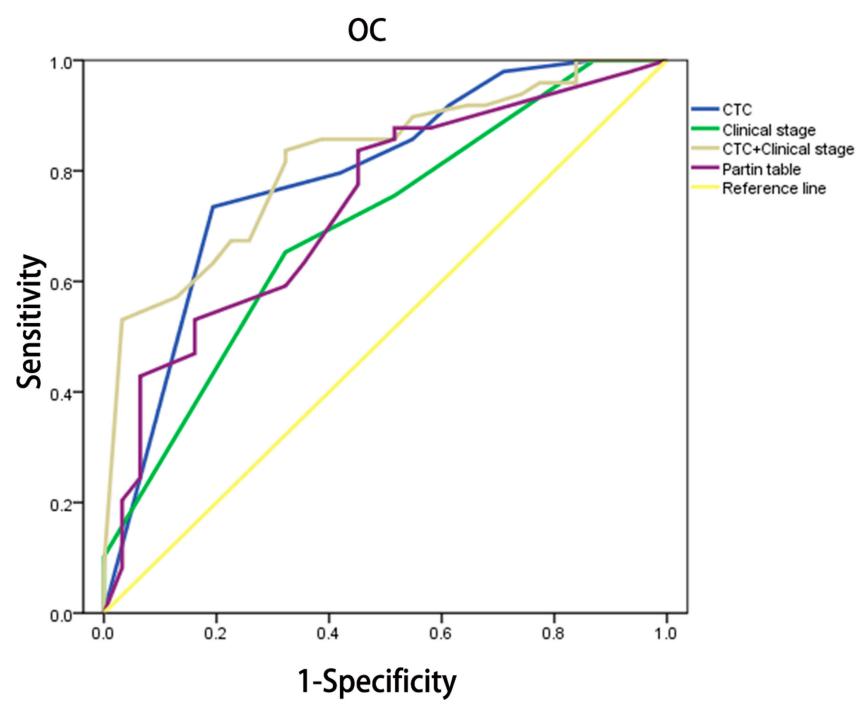

B

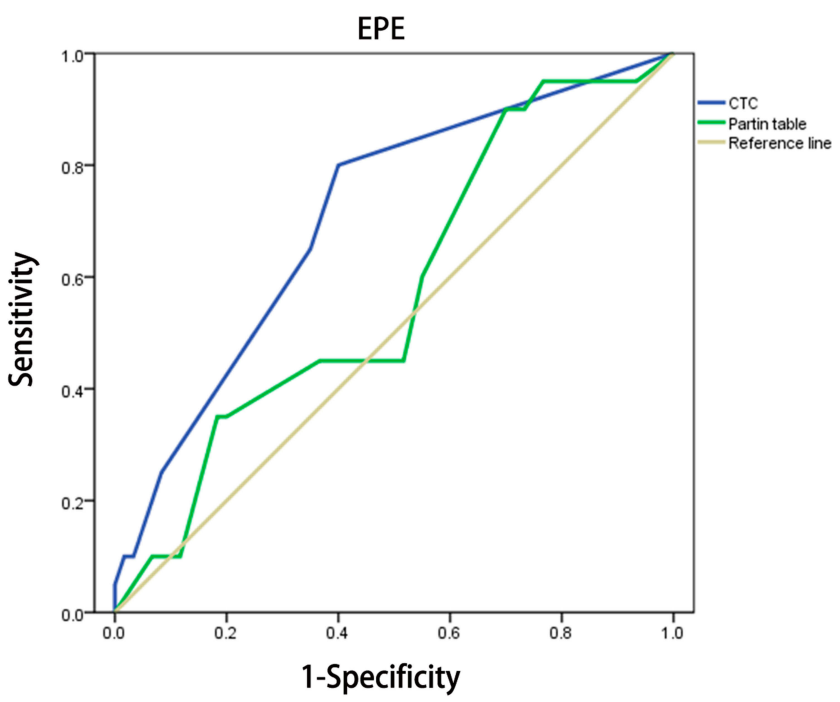

Figure 4 ROC curve analysis on the predictive model of OC and EPE. (A) ROC curve analysis of OC:AUC of CTC combined with clinical stage, clinical stage alone, CTC alone, and Partin table was $0.818,0.698,0.788$, and 0.735 , respectively. (B) ROC curve analysis of EPE:AUC of CTC and Partin table was 0.72 and 0.578 , respectively. Abbreviations: OC, organ-confined; EPE, extracapsular extension. 
Table 5 Logistic Regression Analysis of EPE

\begin{tabular}{|l|l|l|l|l|}
\hline \multirow{2}{*}{ Characteristics } & \multicolumn{2}{|l|}{ Univariate Logistic Regression } & \multirow{2}{*}{ B } & \multicolumn{2}{l|}{ Multivariate Logistic Regression } \\
\cline { 2 - 3 } & HR (95\% CI) & P value & & HR (95\% CI) \\
\hline Age & $1.000(0.98 I-1.020)$ & 0.979 & & \\
Clinical stage & $1.329(0.832-2.124)$ & 0.234 & & \\
PSA level & $1.000(0.981-1.020)$ & 0.979 & & \\
Prostate volume & $1.004(0.979-1.030)$ & 0.760 & & \\
Biopsy Gleason score & $0.831(0.533-1.296)$ & 0.414 & & \\
D'Amico risk classification & $1.146(0.493-2.663)$ & 0.752 & & \\
Total CTCs & $1.197(1.024-1.398)$ & $\mathbf{0 . 0 2 4}$ & & \\
Epithelial CTCs & $1.335(0.941-1.895)$ & 0.105 & & \\
Biophenotypic CTCs & $0.57(0.426-0.762)$ & $\mathbf{0 . 0 0 0}$ & 0.324 & $\mathbf{0 . 0 0 8}$ \\
Mesenchymal CTCs & $0.582(0.184-1.844)$ & 0.358 & & \\
\hline
\end{tabular}

Note: $P$ value $<0.05$ is highlighted in bold.

Abbreviation: EPE, extraprostatic extension, but seminal vesicle invasion and lymph node invasion excluded.

In researches on late stage $\mathrm{PCa}$, the mesenchymal CTCs were the most common and had close association with cancer progression, regarded as the most promising biomarker to assess early development of metastases and therapeutic responses. ${ }^{51}$ In line with previous reports, the mesenchymal subtype in our study had a correlation with seminal vesicle invasion and pathological stage. But no association with PSA, Gleason score, or lymph node invasion was found (Table 3). In the multiple logistic regression analysis on SVI, the mesenchymal subtype showed the highest hazard ratio than clinical stage and biopsy Gleason score Table 2S), in agreement with the notion that the mesenchymal CTCs have a strong metastatic potential. ${ }^{52}$ But as our study focused on localized PCa, the mesenchymal CTCs were detected in merely ten patients with a maximum of three cells. The relatively low detection rate may be the potential reason for limited clinical value in this study. Above all, we demonstrated that the biophenotypic phenotype, but not the mesenchymal or epithelial subtype, was more suitable for localized PCa.

The wide variation of the Partin table makes it unstable to predict stage before surgery in other populations except the US. The EMT subtype CTCs were novel clinical parameters. We intended to add CTCs to traditional clinical variables to build a new approach to predict pathological stage. Through logistic regression, the biophenotypic CTCs as well as clinical stage were finally screened out that associated with OC. The AUC of this prediction model was 0.818 , which was superior to the Partin table 2017 (Figure 4). The AUC of the EPE prediction model was elevated to from 0.578 to 0.72 . But this prediction model has not been verified in other populations. Above all, the EMT CTCs showed promising application value, while larger-scale researches are needed for further improvement.

In summary, our prospective study showed that the biophenotypic CTCs, rather than the epithelial or mesenchymal subtypes, were closely correlated with PSA, Gleason score, and pathological stage in localized PCa. Given its potential to assist in tumor staging, the biophenotypic CTCs were added to a model to predict organ-confined disease before surgery, which was proved superior to the Partin table 2017 in our cohort. This study highlights the clinical impact of the biophenotypic CTCs in localized PCa.

However, there were some limitations in this study. CTCs were detected only once in each patient. Besides, it was a singe center research with a cohort of 80 people. A multicenter, larger-scale cohort study is needed to further verify our conclusion and improve the predictive accuracy.

\section{Data Sharing Statement}

No individual deidentified participant data to be shared.

\section{Acknowledgments}

This work was supported by the Shanghai Shenkang Three Year Action Project (grant no. 16CR2030B).

\section{Disclosure}

The authors report no conflicts of interest in this work.

\section{References}

1. Zhu Y, Freedland SJ, Ye D. Prostate cancer and prostatic diseases best of Asia, 2019: challenges and opportunities. Prostate Cancer Prostatic Dis. 2019. doi:10.1038/s41391-019-0193-7 
2. Aizer AA, Gu X, Chen MH, et al. Cost implications and complications of overtreatment of low-risk prostate cancer in the United States. J Natl Compr Canc Netw. 2015;13(1):61-68. doi:10.6004/ jnccn.2015.0009

3. Fontanella P, Benecchi L, Grasso A, et al. Decision-making tools in prostate cancer: from risk grouping to nomograms. Minerva Urol Nefrol. 2017;69(6):556-566.

4. Tosoian JJ, Chappidi M, Feng Z, et al. Prediction of pathological stage based on clinical stage, serum prostate-specific antigen, and biopsy gleason score: partin tables in the contemporary era. BJU Int. 2017;119(5):676-683. doi:10.1111/bju.13573

5. Xiao W-J, Ye D-W, Yao X-D, Zhang S-L, Dai B. Comparison of three versions of partin tables to predict final pathologic stage in a Chinese cohort: a decision curve analysis. Urol Int. 2013;91 (1):69-74. doi:10.1159/000347186

6. Bhojani N, Ahyai S, Graefen M, et al. Partin tables cannot accurately predict the pathological stage at radical prostatectomy. Eur J Surg Oncol. 2009;35(2):123-128. doi:10.1016/j.ejso.2008.07.013

7. Gupta RT, Brown AF, Silverman RK, et al. Can radiologic staging with multiparametric MRI enhance the accuracy of the partin tables in predicting organ-confined prostate cancer? AJR Am J Roentgenol. 2016;207(1):87-95. doi:10.2214/AJR.15.15878

8. Heitzer E, Haque IS, Roberts CES, Speicher MR. Current and future perspectives of liquid biopsies in genomics-driven oncology. Nat Rev Genet. 2019;20(2):71-88. doi:10.1038/s41576-018-0071-5

9. Scher HI, Heller G, Molina A, et al. Evaluation of circulating tumor cell (CTC) enumeration as an efficacy response biomarker of overall survival (OS) in metastatic castration-resistant prostate cancer (mCRPC): planned final analysis (FA) of COU-AA-301, a randomized, double-blind, placebo-controlled, Phase III study of abiraterone acetate (AA) plus low-dose prednisone (P) post docetaxel. J Clin Oncol. 2011;29(18_suppl):LBA4517-LBA4517.

10. Goldkorn A, Ely B, Quinn DI, et al. Circulating tumor cell counts are prognostic of overall survival in SWOG S0421: a phase III trial of docetaxel with or without atrasentan for metastatic castration-resistant prostate cancer. J Clin Oncol. 2014;32 (11):1136-1142. doi:10.1200/JCO.2013.51.7417

11. Pantel K, Hille C, Scher HI. Circulating tumor cells in prostate cancer: from discovery to clinical utility. Clin Chem. 2019;65 (1):87-99. doi:10.1373/clinchem.2018.287102

12. Meyer CP, Pantel K, Tennstedt P, et al. Limited prognostic value of preoperative circulating tumor cells for early biochemical recurrence in patients with localized prostate cancer. Urol Oncol. 2016;34 (5):235.e211-236. doi:10.1016/j.urolonc.2015.12.003

13. Davis JW, Nakanishi H, Kumar VS, et al. Circulating tumor cells in peripheral blood samples from patients with increased serum prostate specific antigen: initial results in early prostate cancer. $J$ Urol. 2008;179 (6):2187-2191; discussion 2191. doi:10.1016/j.juro.2008.01.102

14. Barrière G, Tartary M, Rigaud M. Epithelial mesenchymal transition: a new insight into the detection of circulating tumor cells. ISRN Oncol. 2012;2012(undefined):382010.

15. Gonzalez DM, Medici D. Signaling mechanisms of the epithelial-mesenchymal transition. Sci Signal. 2014;7(344):re8-re8. doi:10.1126/scisignal.2005189

16. Barriere G, Fici P, Gallerani G, Fabbri F, Zoli W, Rigaud M. Circulating tumor cells and epithelial, mesenchymal and stemness markers: characterization of cell subpopulations. Ann Transl Med. 2014;2(11):109. doi:10.3978/j.issn.2305-5839.2014.10.04

17. Satelli A, Mitra A, Brownlee Z, et al. Epithelial-mesenchymal transitioned circulating tumor cells capture for detecting tumor progression. Clini Cancer Res. 2015;21(4):899-906. doi:10.1158/1078-0432. CCR-14-0894

18. Ni C, Shen Y, Fang Q, et al. Prospective study of the relevance of circulating tumor cell status and neoadjuvant chemotherapy effectiveness in early breast cancer. Cancer Med. 2020;9(7):2290-2298.
19. Zhao R, Cai Z, Li S, et al. Expression and clinical relevance of epithelial and mesenchymal markers in circulating tumor cells from colorectal cancer. Oncotarget. 2017;8(6):9293-9302. doi:10.18632/ oncotarget. 14065

20. Jin X-R, Zhu L-Y, Qian K, et al. Circulating tumor cells in early stage lung adenocarcinoma: a case series report and literature review. Oncotarget. 2017;8(14):23130-23141. doi:10.18632/oncotarget.15506

21. Pan L, Yan G, Chen W, Sun L, Wang J, Yang J. Distribution of circulating tumor cell phenotype in early cervical cancer. Cancer Manag Res. 2019;11(undefined):5531-5536. doi:10.2147/CMAR. S198391

22. Wu S, Liu S, Liu Z, et al. Classification of circulating tumor cells by epithelial-mesenchymal transition markers. PLoS One. 2015;10(4): e0123976. doi:10.1371/journal.pone.0123976

23. Khetani S, Mohammadi M, Nezhad AS. Filter-based isolation, enrichment, and characterization of circulating tumor cells. Biotechnol Bioeng. 2018;115(10):2504-2529. doi:10.1002/bit.26787

24. Awe JA, Saranchuk J, Drachenberg D, Mai S. Filtration-based enrichment of circulating tumor cells from all prostate cancer risk groups. Urol Oncol. 2017;35(5):300-309. doi:10.1016/j.urolonc.2016.12.008

25. Broncy B, Paterlini-Brechot P. Clinical impact of circulating tumor cells in patients with localized prostate cancer. Cells. 2019;8(7):676. doi:10.3390/cells8070676

26. Mottet N, Bellmunt J, Bolla M, et al. EAU-ESTRO-SIOG guidelines on prostate cancer. part 1: screening, diagnosis, and local treatment with curative intent. Eur Urol. 2017;71(4):618-629. doi:10.1016/j. eururo.2016.08.003

27. Li T-T, Liu H, Li FP, et al. Evaluation of epithelial-mesenchymal transitioned circulating tumor cells in patients with resectable gastric cancer: relevance to therapy response. World $J$ Gastroenterol. 2015;21(47):13259-13267. doi:10.3748/wjg.v21.i47.13259

28. Liu Y-K, Hu B-S, Li Z-L, He X, Li Y, Lu L-G. An improved strategy to detect the epithelial-mesenchymal transition process in circulating tumor cells in hepatocellular carcinoma patients. Hepatol Int. 2016;10(4):640-646. doi:10.1007/s12072-016-9732-7

29. Touijer K, Scardino PT. Nomograms for staging, prognosis, and predicting treatment outcomes. Cancer. 2009;115(S13):3107-3111. doi:10.1002/cncr.24352

30. Shen X-C, Qiu Q, Zheng Y-C, Zhang Z. Are partin tables suitable for Chinese patients with prostate cancer? Chin Med J. 2012;125 (21):3795-3799.

31. Bhojani N, Salomon L, Capitanio U, et al. External Validation of the updated partin tables in a cohort of French and Italian men. int $j$ radiat oncol biol phys. 2009;73(2):347-352. doi:10.1016/j. ijrobp.2008.04.082

32. Marrugo-Ramirez J, Mir M, Samitier J. Blood-based cancer biomarkers in liquid biopsy: a promising non-invasive alternative to tissue biopsy. Int J Mol Sci. 2018;19(10):2877. doi:10.3390/ijms19102877

33. Okegawa O, Nutahara N, Higashihara H. Preoperative nested reverse transcription-polymerase chain reaction for prostate specific membrane antigen predicts biochemical recurrence after radical prostatectomy. BJU Int. 1999;84(1):112-117. doi:10.1046/j.1464410x.1999.00076.x

34. Gewanter RM, Katz AE, Olsson CA, et al. RT-PCR for PSA as a prognostic factor for patients with clinically localized prostate cancer treated with radiotherapy. Urology. 2003;61(5):967-971. doi:10.1016/S0090-4295(02)02581-5

35. Helo P, Cronin AM, Danila DC, et al. Circulating prostate tumor cells detected by reverse transcription-PCR in men with localized or castration-refractory prostate cancer: concordance with CellSearch assay and association with bone metastases and with survival. Clin Chem. 2009;55(4):765-773. doi:10.1373/clinchem.2008.117952

36. Gorges TM, Tinhofer I, Drosch M, et al. Circulating tumour cells escape from EpCAM-based detection due to epithelial-tomesenchymal transition. BMC Cancer. 2012;12(1):178. doi:10.1186/ 1471-2407-12-178 
37. Tsumura H, Satoh T, Ishiyama H, et al. Perioperative search for circulating tumor cells in patients undergoing prostate brachytherapy for clinically nonmetastatic prostate cancer. Int J Mol Sci. 2017;18 (1): 128 .

38. Khurana KK, Grane R, Borden EC, Klein EA. Prevalence of circulating tumor cells in localized prostate cancer. Curr Urol. 2013;7 (2):65-69. doi:10.1159/000356251

39. Loh J, Jovanovic L, Lehman M, et al. Circulating tumor cell detection in high-risk non-metastatic prostate cancer. J Cancer Res Clin Oncol. 2014;140(12):2157-2162. doi:10.1007/s00432-014-1775-3

40. Pal SK, He M, Wilson T, et al. Detection and phenotyping of circulating tumor cells in high-risk localized prostate cancer. Clin Genitourin Cancer. 2015;13(2):130-136. doi:10.1016/j. clgc.2014.08.014

41. Xu L, Mao X, Guo T, et al. The novel association of circulating tumor cells and circulating megakaryocytes with prostate cancer prognosis. Clin Cancer Res. 2017;23(17):5112-5122. doi:10.1158/ 1078-0432.CCR-16-3081

42. Satelli A, Batth I, Brownlee Z, et al. EMT circulating tumor cells detected by cell-surface vimentin are associated with prostate cancer progression. Oncotarget. 2017;8(30):49329-49337. doi:10.18632/ oncotarget.17632

43. Yang Y-J, Kong Y-Y, Li G-X, Wang Y, Ye D-W, Dai B. Phenotypes of circulating tumour cells predict time to castration resistance in metastatic castration-sensitive prostate cancer. BJU Int. 2019;124 (2):258-267. doi:10.1111/bju.14642

44. Savagner P. Epithelial-mesenchymal transitions: from cell plasticity to concept elasticity. Curr Top Dev Biol. 2015;112(undefined):273-300.
45. Jolly MK, Tripathi SC, Jia D, et al. Stability of the hybrid epithelial/ mesenchymal phenotype. Oncotarget. 2016;7(19):27067-27084. doi:10.18632/oncotarget.8166

46. Jolly MK, Boareto M, Huang B, et al. Implications of the hybrid epithelial/mesenchymal phenotype in metastasis. Front Oncol. 2015;5:155. doi:10.3389/fonc.2015.00155

47. Schmidt JM, Panzilius E, Bartsch HS, et al. Stem-cell-like properties and epithelial plasticity arise as stable traits after transient twist1 activation. Cell Rep. 2015;10(2):131-139. doi:10.1016/j. celrep.2014.12.032

48. Joosse SA, Gorges TM, Pantel K. Biology, detection, and clinical implications of circulating tumor cells. EMBO Mol Med. 2015;7 (1):1-11. doi:10.15252/emmm.201303698

49. Jolly MK, Somarelli JA, Sheth M, et al. Hybrid epithelial/mesenchymal phenotypes promote metastasis and therapy resistance across carcinomas. Pharmacol Ther. 2019;194(undefined):161-184. doi:10.1016/j.pharmthera.2018.09.007

50. Kölbl AC, Jeschke U, Andergassen U. The significance of epithelial-to-mesenchymal transition for circulating tumor cells. Int J Mol Sci. 2016;17(8):1308. doi:10.3390/ijms17081308

51. Liu W, Yin B, Wang X, et al. Circulating tumor cells in prostate cancer: precision diagnosis and therapy. Oncol Lett. 2017;14 (2):1223-1232. doi:10.3892/ol.2017.6332

52. Russo GI, Bier S, Hennenlotter J, et al. Expression of tumour progression-associated genes in circulating tumour cells of patients at different stages of prostate cancer. BJU Int. 2018;122(1):152-159.

\section{Publish your work in this journal}

Cancer Management and Research is an international, peer-reviewed open access journal focusing on cancer research and the optimal use of preventative and integrated treatment interventions to achieve improved outcomes, enhanced survival and quality of life for the cancer patient.
The manuscript management system is completely online and includes a very quick and fair peer-review system, which is all easy to use. Visit http://www.dovepress.com/testimonials.php to read real quotes from published authors. 